Equilibrium in a Market with Sequential Bargaining

Author(s): Ariel Rubinstein and Asher Wolinsky

Source: Econometrica, Vol. 53, No. 5 (Sep., 1985), pp. 1133-1150

Published by: The Econometric Society

Stable URL: http://www.jstor.org/stable/1911015

Accessed: 07/12/2010 12:51

Your use of the JSTOR archive indicates your acceptance of JSTOR's Terms and Conditions of Use, available at http://www.jstor.org/page/info/about/policies/terms.jsp. JSTOR's Terms and Conditions of Use provides, in part, that unless you have obtained prior permission, you may not download an entire issue of a journal or multiple copies of articles, and you may use content in the JSTOR archive only for your personal, non-commercial use.

Please contact the publisher regarding any further use of this work. Publisher contact information may be obtained at http://www.jstor.org/action/showPublisher?publisherCode=econosoc.

Each copy of any part of a JSTOR transmission must contain the same copyright notice that appears on the screen or printed page of such transmission.

JSTOR is a not-for-profit service that helps scholars, researchers, and students discover, use, and build upon a wide range of content in a trusted digital archive. We use information technology and tools to increase productivity and facilitate new forms of scholarship. For more information about JSTOR, please contact support@jstor.org. 


\title{
EQUILIBRIUM IN A MARKET WITH SEQUENTIAL BARGAINING
}

\author{
By Ariel Rubinstein and Asher Wolinsky
}

\begin{abstract}
This paper considers a market where pairs of agents who are interested in carrying out a transaction are brought together by a stochastic process and, upon meeting, initiate a bargaining process over the terms of the transaction. The basic bargaining problem is treated with the strategic approach.

The paper derives the steady state equilibrium agreements; analyzes their dependence on market conditions such as the relative numbers of agents of different types; and discusses their relations with the competitive equilibrium outcome and other results in the search equilibrium literature.
\end{abstract}

\section{INTRODUCTION}

THIS PAPER CONSIDERS a market that operates in the following way. Pairs of agents who have mutual interest in carrying out a transaction are brought together by a stochastic process. When two agents meet, they initiate a bargaining process over the terms of the transaction. If two agents reach an agreement a transaction takes place and they leave the market. Of course, the bargaining positions and hence the agreement reached in any particular meeting will be affected by the conditions prevailing in the market. These will include the chances that each of the negotiating parties have of meeting other partners in the event that the agreement in the current negotiations is delayed; also the expected length of time required to achieve an alternative transaction, and the expected behavior of alternative partners.

The study of such a market mechanism is of interest for two reasons. Firstly, it captures some realistic aspects of the trade in certain specific markets (e.g., asset markets such as housing and some labor markets). Secondly, it contributes to an understanding of the micro-mechanisms of price formation and their role in shaping market outcomes. In both cases, the features studied are largely neglected in the traditional market equilibrium analyses.

The related literature includes the articles by Diamond $[4,5]$, Diamond and Maskin [6], Mortensen [8, 9], and Zusman and Bell [13]. These articles consider markets of the type described above, in which transactions are concluded at pairwise meetings of agents. The major difference between our work and these articles is in the approach to the basic bargaining problem. In the cited articles it is assumed that a meeting is concluded with an instantaneous agreement which divides the associated surplus in an arbitrary predetermined way (when the surplus is assumed to be divided equally, the division rule is, in fact, Nash's axiomatic bargaining solution). In contrast, the present paper treats the basic bargaining problem with the strategic approach (see Rubinstein [10]) which constitutes an attempt to look into the bargaining black-box. This approach explicitly models the time dimension of the bargaining process, describes in detail the bargaining procedure, and justifies the agreement as a perfect equilibrium in 
the resulting game played between the bargaining parties. Thus, the present paper does not compete with the cited literature, but rather complements it by providing a micro-mechanism that seeks to capture, in a direct fashion, the manner in which market conditions may influence negotiations and hence agreements with which these negotiations conclude (see also Shaked and Sutton [11]).

In Sections 2-4 we present the model, define the solution concept (the market equilibrium), and analyze it. There are two types of agents, and transactions are concluded at pairwise meetings of agents of opposite types. We consider only stationary equilibria where all agents of a particular type employ the same semi-stationary strategy (a strategy that prescribes the same bargaining tactics against any potential opponent). A market equilibrium is a pair of such strategies which is protected from deviation of any agent using any strategy (not necessarily semi-stationary) after any possible history of the agent's market life (a perfectness type requirement). The main theorem (Section 4) claims existence and uniqueness of the market equilibrium and characterizes it. In Section 5 we examine the equilibrium agreements.

One observation that is worth mentioning in the introduction concerns the relations between the equilibrium of the present model and competitive equilibrium. Unsurprisingly, the equilibrium outcome in this model is quite different from that which would be the competitive equilibrium if the trading mechanisms assumed here were replaced by the standard concept of a competitive market. Perhaps of more interest is the observation that even as the costs associated with the bargaining and matching processes are made negligible, the equilibrium outcome of the present model does not approach the competitive equilibrium outcome. This point is of interest since the competitive equilibrium concept does not specify a realistic micro-mechanism of the manner in which the competitive price is realized. A standard textbook justification for its use is that under "competitive conditions," such as the insignificance of each individual agent and negligible transaction costs, it provides a good approximation for the outcome that would be achieved with reasonable micro-mechanisms. But, with the quite natural micro-mechanism presented in this paper, the equilibrium outcomes do not converge to the competitive outcome as the frictions in the model are made indefinitely small. This deviation from the competitive equilibrium is closely related to the familiar point made by Diamond [3] concerning the emergence of a monopoly-price equilibrium in a market where the (numerous) sellers set the prices and the buyers' search is costly. Since in the present model the agents of both types are as likely to be in a position to "set prices" the nature of the deviation from the competitive equilibrium is different from that in Diamond's asymmetric model.

\section{THE MODEL}

The agents in the model are of two different types denoted by 1 and 2. All agents of a particular type are identical. When two agents of opposite types are matched, they bargain over the partition of a unit surplus associated with the 
match. After two agents reach an agreement they leave the market. The model will focus on steady state situations in which this flow of departure is matched by an equal arrival flow of new agents of both types.

This framework can be interpreted in several ways. For example, the two types can be viewed as sellers and buyers of a good. Each seller has one unit for sale and each buyer is seeking to buy one unit. The surplus to be divided is then the difference between their respective reservation prices and an agreement amounts to the determination of the price. Alternatively, one may think of a two-good exchange economy. A trader of type 1 has the initial endowment $(1,0)$ and a trader of type 2 has $(0,1)$. What has to be decided in a meeting is the point on the contract curve to be reached in the exchange.

The market considered in the model operates over time. The time dimension is discrete. The time periods in the market life of an agent will be labelled with a nonnegative integer $t$ where $t=0$ is the time period at which the agent in question arrives in the market. No upper limit is placed on the value of $t$ which corresponds to the assumption that, after arrival, he may remain indefinitely in the market. The two processes to be modelled which depend on time are matching and bargaining.

\section{The Matching Process}

At the beginning of each time period there is a matching stage in which each agent may meet at most one new partner (the term "new partner" refers to a member of the opposite type with whom the agent did not bargain in the previous period). At each matching stage the probability for an agent of type 1 (2) to meet a new partner is $\alpha(\beta)$, where $0<\alpha, \beta \leqslant 1$. The parameters $\alpha$ and $\beta$ are assumed fixed over time.

The last assumption has to do with our intention to focus on a market at a steady state. We think of $\alpha$ and $\beta$ as functions of $N_{1}$ and $N_{2}\left(N_{i}\right.$ is the size of the population of type $i$ ) and the meeting technology which is not modeled explicitly. At a steady state $N_{1}$ and $N_{2}$ are constant over time and therefore $\alpha$ and $\beta$ are constant as well.

REMARK: To understand what we mean by a steady state, observe that given $N_{1}$ and $N_{2}, \alpha=\alpha\left(N_{1}, N_{2}\right)$ and $\beta=\beta\left(N_{1}, N_{2}\right)$ determine the rate at which matches are formed. At the equilibrium which is discussed later, $\alpha$ and $\beta$ will also determine the rate at which agreements are reached and agents leave the markets. We assume that there is an exogenous flow of agents into the market whereby in each period equal numbers of both types are added (if the added number were not equal, then one of the populations would be growing without a limit relative to the other). At a steady state $N_{1}$ and $N_{2}$ are such that the equilibrium flow of departures which is determined by $\alpha=\alpha\left(N_{1}, N_{2}\right)$ and $\beta=\beta\left(N_{1}, N_{2}\right)$ is exactly equal to the exogenous flow of arrivals. After we present the equilibrium the reader will be able to verify that if we adopt, for example, a linear meeting technology whereby the number of matches in each period is $k_{1} N_{1}+k_{2} N_{2}$, then 
for any exogenous flow of arrivals and any initial difference $d$ there exist $N_{1}$ and $N_{2}$ of steady state such that $N_{2}-N_{1}=d$.

\section{The Bargaining Process}

At a given time period an agent can bargain with at most one partner. The bargaining between two matched agents need not be concluded in one period. In the wake of a matching stage, an agent can be in one of the following three states: he has no partner; he has a new partner; he has a partner with whom he was already in the process of bargaining in the previous period. In either one of the latter two cases the parties proceed to the bargaining stage.

The bargaining procedure is the same for all the time periods. Firstly, one of the parties is selected randomly, with probability $\frac{1}{2}$ to propose a partition of the unit to which proposal the other party then reacts with acceptance (" $Y$ ") or rejection (" $N$ "). Acceptance of a proposal ends the bargaining and both parties leave the market. If a proposal is rejected, then the same bargaining procedure is repeated in the next time period unless the matching process assigns one or both of the parties a new bargaining partner for the next period.

The bargaining procedure adopted here is a modified version of the alternating offers procedure studied by Rubinstein [10] and Stahl [12]. This version was studied by Binmore [1] under the heading "random selection of proposers."

\section{The Order of Events}

As is evident from the above description, the processes of matching and bargaining are interlaced. Let us present the exact connections between them by describing the order of events for a typical agent of type 1 at a certain time period (an analogous description will suit an agent of type 2). First, the matching stage takes place. If the agent was not in the process of bargaining in the previous period, then with probability $\alpha$ he meets a new partner with whom he proceeds to the bargaining stage, and with probability $1-\alpha$ he remains without a partner. If the agent was in the process of bargaining in the previous period and did not reach an agreement, then there are three possible cases: with probability $\alpha$ he meets a new partner and regardless of what happened to his previous partner he abandons him and starts bargaining with the new one; with probability $(1-\alpha)(1-\beta)$ neither the agent nor his previous partner meet new partners and they continue their bargaining in this period as well; with probability $(1-\alpha) \beta$ the agent does not meet a new partner but his old partner does and so the agent remains without a partner until matched again at some future period.

Notice that we assume that, whenever an agent meets a new partner, he abandons the old one. It will become evident that, at the equilibrium to be discussed later, this assumption is consistent with rational behavior. What we have in mind is a situation where the arrival of a new partner occurs before the 
agent knows whether his old partner is still available and therefore the assumed choice is natural. ${ }^{1}$ The purpose of this assumption is to capture the idea that, when a bargaining process is dragged on, each party runs the risk of losing the deal and having to wait again for another opportunity. This risk creates a pressure on the bargaining parties to reach an agreement. Of course, this point can be captured by somewhat different assumptions as well (say, probabilistic choice between an old and a new partner). But the reader can verify that such modifications will not alter the qualitative nature of the equilibrium results.

\section{Preferences}

An agent may leave the market $t$ periods after entering it with a fraction $z$ of the unit surplus $(0 \leqslant z \leqslant 1, t \geqslant 0)$, or else he may continue meeting and bargaining without ever reaching an agreement. All agents are assumed to be maximizers of expected utility. The utility of getting a fraction $z$ at time $t$ is the same for both types of agents and is given by $\delta^{t} z$, where $\delta<1$ is a discount factor. A utility of 0 is assigned to an agent who never leaves the market.

We chose the form $\delta^{t} z$ mainly for the sake of simplicity. It seems, however, that the discussion can be extended to a much wider family of time preferences. ${ }^{2}$

\section{Histories}

It is assumed that the agents have perfect recall. A possible history of an agent at a certain stage of his market life is a sequence of all the observations made by him up to that stage. During any period, $T$, the agent receives the relevant information sequentially. That is, he gets one by one the answers to the following questions in the following order: (I) Does he have a partner at period $T$ ? If so, did he have this same partner at period $T-1$ ? (II) Who was selected to make an offer at $T$ ? (III) What was the offer at period $T$ ? (IV) What was the response to that offer? This information is added to the information accumulated until the end of period $T-1$. Thus, a possible history at a certain stage in period $T$ is a sequence of answers to the above questions in all the previous periods and up to that stage at period $T$.

We shall be particularly interested in the histories after which the agents have to make decisions. Let $H_{o i}^{T}$ be the set of all possible histories which end with the information that, at period $T$, the agent has a partner and that the bargainer of

\footnotetext{
${ }^{1}$ We have a continuous time version of this model in which a new partner can arrive at any point in the interval of time elapsed between consecutive stages in an ongoing bargaining process. In that version, the assumed choice is obvious since the arrival of a new partner strictly precedes the resumption of the bargaining. We chose not to present that version since it yields the same results while it is more complicated.

${ }^{2}$ We think that the discussion can be extended to the family of time preferences over the pairs $(x, t)$ representable by $\delta^{t} u(x)$. As shown by Fishburn and Rubinstein [7] this is the family of all monotonic, continuous, and stationary time preferences.
} 
type $i(i=1,2)$ was selected to make an offer. ${ }^{3}$ Let $H_{R i}^{T}$ be the set of all possible histories which end at period $T$ with the information about the offer to which the bargainer of type $i(i=1,2)$ has to respond.

Finally, notice that an agent remembers the identity of his partner only as long as they are in the process of bargaining (see question I above). He does not remember, however, the identities of those with whom he had bargained in the past (and broken off). That is, if he happens to meet again an agent with whom he had already bargained and broken off, he treats him as a new partner.

\section{Strategies}

In what follows a partition of the unit surplus, $(x, 1-x)$, will be identified with the fraction, $x$, received by the agent of type 1 . That is, saying that parties to a bargaining process agree on the partition $x$ amounts to saying that the agent of type 1 receives $x$ and his partner receives $1-x$.

A strategy for an agent of type $1, f \in S_{1}$, is a sequence of decision rules, $f=\left(f^{t}\right)_{t=0}^{\infty}$. The $t$ th decision rule, $f^{t}$, describes the agent's behavior conditional on the information he will have gathered until he has to make a decision at period $t$. That is, $f^{t}$ is a function defined over all the histories in $H_{01}^{t}$ and $H_{R 1}^{t}$. If $h^{t} \in H_{01}^{t}$, then the agent (who is of type 1) has to make an offer and $f^{t}\left(h^{t}\right) \in[0,1]$. If $h^{t} \in H_{R 1}^{t}$, then the agent has to respond to an offer and $f^{t}\left(h^{t}\right) \in\{$ “" $Y$ ", “ $N$ " $\}$. Finally, a strategy for an agent of type 2 can be analogously defined, and will be denoted by $g \in S_{2}$.

In the sequel we shall sometimes refer to a special type of strategy. A semistationary strategy prescribes the same bargaining tactics against all the partners that the agent might meet. That is, the length of the waiting periods, and the nature and number of the unsuccessful bargaining processes that the agent has gone through do not affect his bargaining strategy against a new partner. More formally, let $h^{t}$ and $h^{t^{\prime}}$ be two histories such that the last piece of information in each is that the agent has just met a new partner. A semi-stationary strategy, $f$, is such that, for any two histories $h^{T}$ and $h^{T^{\prime}}\left(T-t=T^{\prime}-t^{\prime}\right)$, which coincide with $h^{t}$ and $h^{t^{\prime}}$ in their earlier parts and are identical thereafter, we have $f^{T}\left(h^{T}\right)=f^{T^{\prime}}\left(h^{T^{\prime}}\right)$. Let $\bar{S}_{i}$ denote the set of all semi-stationary strategies for an agent of type $i$.

\section{The Equilibrium}

Given the strategy $f \in S_{1}$ and semi-stationary strategy $g \in \bar{S}_{2}$, let $U_{1}(f, g)\left(h^{T}\right)$ be the value at time $T$ of the expected utility to an agent of type 1 who has

\footnotetext{
${ }^{3}$ Notice that the set $H_{0 i}^{T}$ includes personal histories of both type $i$ and type $j \neq i$ agents. Thus, when a personal history of a type 1 agent belongs to $H_{01}^{T}$, it ends with the information that, at period $T$, this agent himself was selected to make an offer; when a personal history of a type 2 agent belongs to $H_{01}^{T}$, it ends with the information that, at period $T$, this agent's opponent was selected to make an offer and hence the agent himself has no decision to make at this point. An analogous remark applies for the set $H_{R i}^{T}$ defined later in the paragraph.
} 
experienced the history $h^{T}$, and who employs the strategy $f \in S_{1}$ while all his potential partners employ the semi-stationary strategy $g \in \bar{S}_{2}$ (the possibility that the agent reaches an agreement $x$ at time $t \geqslant T$ appears in $U_{1}(f, g)\left(h^{T}\right)$ as the term $\delta^{t-T} x$ weighted by the appropriate probability). Notice that $U_{1}(f, g)\left(h^{T}\right)$ depends on two sequences of independent random variables. One sequence determines the rate at which new matches are formed and old matches break up (given the parameters $\alpha, \beta$ ), while the other sequence determines the proposer at each bargaining stage. Notice further that for $U_{1}(f, g)\left(h^{T}\right)$ to be meaningful it is essential that $g$ be semi-stationary. If $g$ were not semi-stationary, the histories of the agent's partners could be important as well, but since they are not known to the agent, $U_{1}(f, g)\left(h^{T}\right)$ would have little meaning. Similarly, given $g \in S_{2}$ and $f \in \bar{S}_{1}$ one can define $U_{2}(f, g)\left(h^{T}\right)$.

We are now ready to define the market equilibrium.

DEFINITION: A market equilibrium is a pair of semi-stationary strategies $\left(f_{*}, g_{*}\right)$ such that all agents of type 1 employ strategy $f_{*}$, all agents of type 2 employ strategy $g_{*}$, and for all possible histories $h^{T}$,

$$
\begin{array}{lll}
U_{1}\left(f_{*}, g_{*}\right)\left(h^{T}\right) \geqslant U_{1}\left(f, g_{*}\right)\left(h^{T}\right) & \text { for all } & f \in S_{1}, \\
U_{2}\left(f_{*}, g_{*}\right)\left(h^{T}\right) \geqslant U_{2}\left(f_{*}, g\right)\left(h^{T}\right) & \text { for all } & g \in S_{2} .
\end{array}
$$

Thus, in looking for a market solution we restrict our attention to configurations where all agents employ semi-stationary strategies and where all agents of a certain type use the same strategy. Without this restriction we cannot analyze the bargaining process as bargaining with complete information and will face instead the much more complicated problem of bargaining with incomplete information and probably with ignorance. Notice, however, that the equilibrium is protected against any possible deviation $f \in S_{1}$ (not necessarily semi-stationary). Finally, observe that the equilibrium has the flavor of Selten's subgame perfectness. Given any history $h^{T}$, including histories which are not consistent with $f_{*}$, an agent cannot improve his expected payoff by deviating from his equilibrium strategy.

\section{A BARGAINING GAME WITH RANDOM SELECTION OF PROPOSERS}

The bargaining between two matched agents is an important component of the model. In order to analyze it we present in this section a sequential bargaining game and derive its perfect equilibrium. In a later section we shall incorporate this game into the model and make the necessary adjustments to identify it with the bargaining that goes on in the considered market.

The game is played by two players, 1 and 2, who bargain over the partition of a unit. The bargaining process takes place over time, and the discrete time periods will be denoted by $t, t=0,1,2,3, \ldots$ In each period one of the players is selected randomly (with probability $\frac{1}{2}$ and independently of previous selections) to propose a partition of the unit. The other player has to react to the offer in the same period by accepting it- " $Y$ "-or rejecting it- " $N$ ". If a partition $x$ is agreed 
upon at time $t$, then the game ends and the outcome is described as $(x, t)$ (recall that partition $x$ means that player 1 receives the fraction $x$ and player 2 receives the fraction $1-x)$.

Let $u_{i}(z, t)$ denote the utility to player $i$ from getting the fraction $z$ at time $t$. Thus, according to our notational convention the utilities associated with the outcome $(x, t)$ are $u_{1}(x, t)$ and $u_{2}(1-x, t)$. It is assumed that, for all $z, u_{i}(z, 0)=z$ and $u_{i}(z, t) \in[0,1]$. It is further assumed that $u_{i}(z, t)$ and $z-u_{i}(z, t)$ are strictly increasing in $z$, that the utility of perpetual nonagreement is $u_{i}(0, \infty)=0$, and that $u_{i}(z, t)$ is stationary over time. ${ }^{4}$ The stationarity assumption means that $u_{i}(z, 0) \geqslant u_{i}\left(z^{\prime}, 1\right)$ if and only if $u_{i}(z, t) \geqslant u_{i}\left(z^{\prime}, t+1\right)$ for all $t$. Finally, it is assumed that the players are expected utility maximizers.

A strategy in this game, $f=\left(f^{t}\right)_{t=0}^{\infty}$, has the same structure as the strategies defined in Section 2. The definition of a history, $h^{t}$, is slightly different here. In this game there are only three pieces of information collected each period. These are the answers to questions II, III, IV in the definition of a history in Section 2.

The following proposition deals with existence, uniqueness, and characterization of the perfect equilibrium of the considered bargaining game. This proposition is analogous to the main theorem in Rubinstein [10] and is similar to a result in Binmore [1]. For the sake of brevity we omit the proof. ${ }^{5}$

\section{Proposition 1: The system}

$$
\begin{aligned}
& y=\frac{1}{2} u_{1}(x, 1)+\frac{1}{2} u_{1}(y, 1), \\
& 1-x=\frac{1}{2} u_{2}(1-y, 1)+\frac{1}{2} u_{2}(1-x, 1),
\end{aligned}
$$

has a unique solution $\left(x_{*}, y_{*}\right)$ such that $y_{*} \leqslant x_{*}$, and the unique perfect equilibrium (P.E.) of the game, $\left(f_{*}, g_{*}\right)$, is

$$
\begin{aligned}
& f_{*}^{t}\left(h^{t}\right)= \begin{cases}x_{*}, & h^{t} \in H_{01}^{t}, \\
" Y ", & h^{t} \in H_{R 1}^{t} \text { and the last proposal was } y \geqslant y_{*}, \\
" N ", & h^{t} \in H_{R 1}^{t} \text { and the last proposal was } y<y_{*} ;\end{cases} \\
& g_{*}^{t}\left(h^{t}\right)= \begin{cases}y_{*}, & h^{t} \in H_{02}^{t}, \\
“ Y ”, & h^{t} \in H_{R 2}^{t} \text { and the last proposal was } x \leqslant x_{*}, \\
" N ", & h^{t} \in H_{R 2}^{t} \text { and the last proposal was } x>x_{*} .\end{cases}
\end{aligned}
$$

ReMARK: Notice that the meaning of system (1) is that $x_{*}$ and $y_{*}$ are such that player 1 is just indifferent between accepting $y_{*}$ or rejecting it and then getting with equal probabilities $x_{*}$ and $y_{*}$ in the next period. Similarly, player 2

\footnotetext{
${ }^{4}$ For the purposes of this paper we need only consider functions $u_{t}(z, t)$ such that $u_{i}(z, 1)=a_{i}+b_{i} z$, where $0 \leqslant b_{i}<1$. We treat here the more general case not only because it is more general but also because in doing so we simplify the required notation.

${ }^{5}$ The proof appears in an earlier version of this paper; see D.P. 83/63, ICERD, London School of Economics.
} 
is just indifferent between getting $1-x_{*}$ this period, or, rejecting it and getting $1-x_{*}$ or $1-y_{*}$ in the next period.

\section{MARKET EQUILIBRIUM}

This section contains the main theorem that deals with existence, uniqueness, and characterization of the market equilibrium.

THEOREM: There exists a unique market equilibrium (M.E.), $\left(f_{*}, g_{*}\right)$. The equilibrium strategies are

$$
\begin{aligned}
& f_{*}\left(h^{t}\right)= \begin{cases}x_{*}, & h^{t} \in H_{01}^{t}, \\
“ Y ”, & h^{t} \in H_{R 1}^{t} \text { and the last offer was } y \geqslant y_{*}, \\
“ N^{\prime}, & \text { otherwise; }\end{cases} \\
& g_{*}\left(h^{t}\right)= \begin{cases}y_{*}, & h^{t} \in H_{02}^{t}, \\
“ Y ”, & h^{t} \in H_{R 2}^{t} \text { and the last proposal was } x \leqslant x_{*}, \\
\text { “N”, } & \text { otherwise; }\end{cases}
\end{aligned}
$$

where

$$
\begin{aligned}
& x_{*}=\frac{2(1-\delta)+\delta \alpha-\delta(1-\delta)(1-\alpha)(1-\beta)}{2(1-\delta)+\delta \alpha+\delta \beta}, \\
& y_{*}=\frac{\delta \alpha+(1-\delta) \delta(1-\alpha)(1-\beta)}{2(1-\delta)+\delta \alpha+\delta \beta} .
\end{aligned}
$$

The rest of this section is devoted to the proof of the theorem (the nature of the equilibrium and the meaning of $x_{*}$ and $y_{*}$ will be discussed in the following section).

Recall that the equilibrium strategies are constrained to be semi-stationary. Since a semi-stationary strategy is such that the same bargaining strategy is used against every partner, it is characterized by a bargaining strategy in the bargaining game described in Section 3. That is, there is one-to-one correspondence between the set of semi-stationary strategies and the set of strategies in the bargaining game of Section 3 . Let $\tilde{f}, \tilde{g}$ denote the strategies in the bargaining game which correspond to the semi-stationary strategies $f \in \bar{S}_{1}, g \in \bar{S}_{2}$.

The proof consists of three claims. Claim 1 shows that every M.E., $(f, g)$, induces a P.E., $(\tilde{f}, \tilde{g})$, in the bargaining game of Section 3 with properly chosen utility functions. Claim 2 uses Proposition 1 to show that the P.E. of the bargaining game is $\left(\tilde{f}_{*}, \tilde{g}_{*}\right)$, where $f_{*}$ and $g_{*}$ are the strategies described in the Theorem. Thus, Claims 1 and 2 prove uniqueness and characterize the M.E. Claim 3 shows that $\left(f_{*}, g_{*}\right)$ is indeed M.E. Thus, Claim 3 proves existence and Claims 1,2 prove uniqueness and characterize the M.E.

Before we turn to the claims we need the following preparations. First, given $f \in S_{1}$ and $g \in \bar{S}_{2}$, we introduce special notation for the expected utility in two 
special cases. Let

$$
V_{1}(f, g)=U_{1}(f, g)\left(h_{-}^{0}\right), \quad W_{1}(f, g)=U_{1}(f, g)\left(h_{+}^{0}\right),
$$

where $h_{-}^{0}$ includes only the information that, at period 0 , the agent does not have a partner, and $h_{+}^{0}$ includes only the information that, at period 0 , he has a partner. Similarly, given $g \in S_{2}$ and $f \in \bar{S}_{1}$, one can define $V_{2}(f, g)$ and $W_{2}(f, g)$.

Next, consider an agent of type 1 and let $h^{T}$ be a history which ends with the information that the agent has a partner at period $T$. Given $f \in S_{1}, g \in \bar{S}_{2}$, the value of $U_{1}(f, g)\left(h^{T}\right)$ can be calculated as follows. First consider the corresponding strategies $\tilde{f}, \tilde{g}$ in the bargaining game. There exists a distribution of outcomes, $(x, t)$, in the bargaining game when it is played with $\tilde{f}, \tilde{g}$ after the relevant bargaining history which is implied by $h^{T}$. Second, let $\tau$ be a random variable which represents the length of time until the breakdown of the current bargaining process. That is, $\tau$ will assume the value $t_{0}$ with the conditional probability that, if no agreement is reached in the next $t_{0}$ periods, the current bargaining will break down at $T+t_{0}$. Therefore, conditional on the fact that no agreement is reached, Prob $\left(\tau>t_{0}\right)=\gamma^{t_{0}}$ where $\gamma=(1-\alpha)(1-\beta)$. The distributions of $(x, t)$ and $\tau$ determine $U_{1}(f, g)\left(h^{T}\right)$ as follows:

$$
\begin{aligned}
U_{1}(f, g)\left(h^{T}\right)= & \sum_{\left(x_{0}, t_{0}\right)} \operatorname{Prob}\left[(x, t)=\left(x_{0}, t_{0}\right)\right]\left\{\delta^{t_{0}} x_{0} \operatorname{Prob}\left(\tau>t_{0}\right)\right. \\
& \left.+\sum_{n=1}^{t_{0}} \delta^{n} \operatorname{Prob}(\tau>n-1)\left[\alpha W_{1}(f, g)+\beta(1-\alpha) V_{1}(f, g)\right]\right\} \\
= & \underset{(x, t)}{E}\left(\gamma^{t} \delta^{t} x\right)+\underset{(x, t)}{E} \sum_{n=1}^{t} \gamma^{n-1} \delta^{n}\left[\alpha W_{1}(f, g)\right. \\
& \left.+\beta(1-\alpha) V_{1}(f, g)\right] .
\end{aligned}
$$

The expectation operator in the last line is, of course, with respect to the distribution of outcomes in the bargaining game played with $\tilde{f}, \tilde{g}$ after the relevant bargaining history of the current partners. Notice that the first term on the r.h.s. accounts for the possibility that the current bargaining process will be concluded successfully, while the second term accounts for the possibility that it will break down. In the latter event the expected utility at the point of the breakdown is either $W_{1}$ or $V_{1}$.

The formula (2) was derived from semi-stationary strategies, but can be extended. To see this, let $f \in \bar{S}_{1}, g \in \bar{S}_{2}$, and $h^{T}$ be as before and let $f^{\prime} \in S_{1}$ be a strategy which might differ from $f$ only after histories which are continuations of $h^{T}$ and include the information that the partner is still the same one this agent of type 1 had at period $T$. That is, $f^{\prime}$ might differ from $f$ only for the duration of a single bargaining process. Now, formula (2) is valid for $U_{1}\left(f^{\prime}, g\right)\left(h^{T}\right)$. Here the expectation operator is with respect to the distribution of outcomes $(x, t)$ in the bargaining game played with the bargaining strategy prescribed by $f^{\prime}$ for the current bargaining process and with $\tilde{g}$. The argument of $V_{1}(f, g)$ and $W_{1}(f, g)$ is still $f$, since $f^{\prime}$ differs from $f$ only during the current bargaining process. Finally, the analogous formula can be derived for $U_{2}(f, g)\left(h^{T}\right)$. 
Claim 1: If $(f, g)$ is a M.E., then $(\tilde{f}, \tilde{g})$ is a P.E. of the bargaining game of Section 3 with utility functions

$$
\begin{aligned}
& u_{1}(z, t)=\gamma^{t} \delta^{t} z+\sum_{n=1}^{t} \gamma^{n-1} \delta^{n}\left[\alpha W_{1}+\beta(1-\alpha) V_{1}\right], \\
& u_{2}(z, t)=\gamma^{t} \delta^{t} z+\sum_{n=1}^{t} \gamma^{n-1} \delta^{n}\left[\beta W_{2}+\alpha(1-\beta) V_{2}\right],
\end{aligned}
$$

where $V_{i}=V_{i}(f, g), W_{i}=W_{i}(f, g)$, and $\gamma=(1-\alpha)(1-\beta)$.

Proof: First notice that $u_{1}$ and $u_{2}$ satisfy the conditions of Section 3. Assume that $(\tilde{f}, \tilde{g})$ is not a P.E. in the bargaining game. Then there exists a history $\tilde{h}^{T}$ in the bargaining game such that one of the players, say player 1, has a profitable deviation $\tilde{f}_{0}$. That is,

$$
\tilde{U}_{1}\left(\tilde{f}_{0}, \tilde{g}\right)\left(\tilde{h}^{T}\right)>\tilde{U}_{1}(\tilde{f}, \tilde{g})\left(\tilde{h}^{T}\right) .
$$

Define a market strategy, $f^{\prime} \in S_{1}$, as follows: when the agent bargains with his first partner he follows $\tilde{f}_{0}$; with any other partner he follows the bargaining strategy $\tilde{f}$. Let $h^{T}$ be the market history of a type 1 agent who, at his first period, met a partner and has since then bargained with him according to the history $\tilde{h}^{T}$.

From (2) and the choice of $u_{1}(z, t)$ we have

$$
U_{1}(f, g)\left(h^{T}\right)=\underset{(z, t)}{E} u_{1}(z, t)=\tilde{U}_{1}(\tilde{f}, \tilde{g})\left(\tilde{h}^{T}\right),
$$

where the last equality follows from the definition of $\tilde{U}_{1}$ and the expectation operator is with respect to the distribution of outcomes in the game with $\tilde{f}, \tilde{g}$ after $\tilde{h}^{T}$. From the choice of $f^{\prime},(3)$ and (4) it follows that

$$
\begin{aligned}
U_{1}\left(f^{\prime}, g\right)\left(h^{T}\right) & =\tilde{U}_{1}\left(\tilde{f}_{0}, \tilde{g}\right)\left(\tilde{h}^{T}\right)>U_{1}(\tilde{f}, \tilde{g})\left(\tilde{h}^{T}\right) \\
& =U_{1}(f, g)\left(h^{T}\right)
\end{aligned}
$$

in contradiction to the assumption that $(f, g)$ is a M.E.

Q.E.D.

Claim 2: If there exists a M.E., it is unique and is given by the pair $\left(f_{*}, g_{*}\right)$ described in the Theorem.

Proof: Let $(f, g)$ be a M.E. By Claim 1 the $\operatorname{pair}(\tilde{f}, \tilde{g})$ is a P.E. of the bargaining game with the utility functions $u_{1}$ and $u_{2}$ described there. By Proposition 1, we have that this P.E. must be the one described there. That is, the P.E. strategies $\tilde{f}$ and $\tilde{g}$ are characterized by numbers $x$ and $y$ which satisfy the equations

$$
\begin{gathered}
y=\frac{1}{2} u_{1}(x, 1)+\frac{1}{2} u_{1}(y, 1)=\frac{1}{2} \gamma \delta(x+y)+\delta\left[\alpha W_{1}+\beta(1-\alpha) V_{1}\right], \\
\begin{aligned}
1-x & =\frac{1}{2} u_{2}(1-y, 1)+\frac{1}{2} u_{2}(1-x, 1) \\
& =\frac{1}{2} \gamma \delta(1-x+1-y)+\delta\left[\beta W_{2}+\alpha(1-\beta) V_{2}\right] .
\end{aligned}
\end{gathered}
$$


With these strategies we have that $W_{i}=W_{i}(f, g)$ are given by

$$
\begin{aligned}
& W_{1}=\frac{1}{2} x+\frac{1}{2} y, \\
& W_{2}=\frac{1}{2}(1-x)+\frac{1}{2}(1-y) .
\end{aligned}
$$

Further, from the definition of $V_{i}=V_{i}(f, g)$ we have

$$
\begin{aligned}
& V_{1}=\delta\left[(1-\alpha) V_{1}+\alpha W_{1}\right], \\
& V_{2}=\delta\left[(1-\beta) V_{2}+\beta W_{2}\right] .
\end{aligned}
$$

Thus, there are six equations with six variables $\left(x, y, V_{1}, V_{2}, W_{1}, W_{2}\right)$ and it is a matter of routine calculations to show that $x$ and $y$ are equal to the values $x_{*}$ and $y_{*}$ given in the Theorem. Therefore, if $(f, g)$ is a M.E., it must coincide with $\left(f_{*}, g_{*}\right)$ of the Theorem.

Q.E.D.

It remains to show that there exists a M.E.

Claim 3: The pair $\left(f_{*}, g_{*}\right)$ described in the Theorem is a M.E.

Proof: Consider an agent of type 1. First we show that, when all type 2 agents employ strategy $g_{*}$, this agent faces a Markov decision problem. Then we use a known result to derive the optimal policy for this Markov decision process. Finally, the optimality of $f_{*}$ will follow from identifying it with the derived optimal policy.

The problem of a type 1 agent is equivalent to the following Markov decision problem. The state space is $\left\{e_{1}, e_{2}, e_{3}, e_{4}\right\}$ where: $e_{1}$ denotes that the agent does not have a partner; $e_{2}$ means the agent has a partner and he has to make an offer; $e_{3}$ represents that the agent has a partner and the partner has offered $y_{*}$; $e_{4}$ means agreement was reached (an absorbing state). The set of actions is $A=\{$ stop, continue $\}$, where the action stop means to reach an agreement (i.e., accept $y_{*}$ or offer $x_{*}$ ). At each time period $t$ this Markov decision process is at state $e^{t} \in\left\{e_{1}, e_{2}, e_{3}, e_{4}\right\}$ and the agent takes an action $a^{t} \in A$. The process starts at initial state $e^{1} \in\left\{e_{1}, e_{2}, e_{3}\right\}$ and proceeds according to the transition probabilities, $\operatorname{Prob}\left(e^{t+1}=e_{3} \mid a^{t}=\operatorname{stop}\right)=1$, and $\operatorname{Prob}\left(e^{t+1}=e_{i} \mid e^{t}=e_{j}, a=\right.$ continue $)$ which are given by the matrix

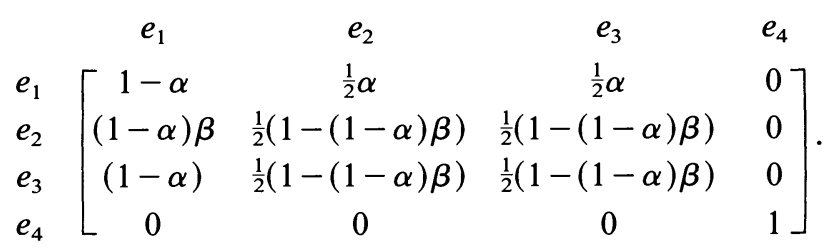


The periodic payoff is

$$
\phi\left(e^{t}, a^{t}\right)= \begin{cases}x_{*}, & e^{t}=e_{1}, \quad a^{t}=\text { stop } \\ y_{*}, & e^{t}=e_{2}, \quad a^{t}=\text { stop }, \\ 0, & \text { otherwise. }\end{cases}
$$

The objective is to find a policy $D, D\left(e^{0}, \ldots, e^{t}, t\right)=a^{t}$, that, given the initial state $e^{0}$, maximizes $\Phi\left(D, e^{0}\right)=E\left[\sum_{t=0}^{\infty} \delta^{t} \phi\left(e^{t}, a^{t}\right)\right]$.

It is well known that for such a Markov decision problem there exists a stationary optimal policy (see Derman [2]). Here there is only a limited number of stationary policies and all of the form: stop whenever $e^{t}$ belongs to a certain subset of $\left\{e_{1}, e_{2}, e_{3}\right\}$. A routine computation will reveal that the following two stationary policies are optimal: $D^{*}=$ stop whenever $e^{t} \in\left\{e_{2}, e_{3}\right\}$, and $D^{\prime}=$ stop only when $e^{t}=e_{2}$. The values are $\Phi\left(D^{*}, e_{1}\right)=\frac{1}{2} \alpha\left(x_{*}+y_{*}\right) /[1-(1-\alpha) \delta]$, $\Phi\left(D^{*}, e_{2}\right)=x_{*}$, and $\Phi\left(D^{*}, e_{3}\right)=y_{*}$.

Now, notice that any strategy $f \in S_{1}$ implies a policy $D$ and that for any history $h^{T}$ ending at one of the states $e_{1}, e_{2}, e_{3}, U_{1}\left(f, g_{*}\right)\left(h^{T}\right)=\Phi\left(D, e^{0}=e_{i}\right)$. Notice further that the optimal policy $D^{*}$ is precisely the behavior prescribed by the strategy $f_{*}$. Therefore, after the history $h^{t} \in H_{01}^{t}$ and $h^{t} \in H_{R 1}^{t}$ where the last offer was $y_{*}$, the strategy $f_{*}$ is optimal. Finally, given $h^{t} \in H_{R 1}^{t}$ where the last offer was $y \neq y_{*}$, the agent has to compare $y$ to

$$
(1-\alpha) \beta \delta \Phi\left(D^{*}, e_{1}\right)+\frac{1}{2}(1-(1-\alpha) \beta) \delta\left[\Phi\left(D^{*}, e_{2}\right)+\Phi\left(D^{*}, e_{3}\right)\right] .
$$

On substituting the values of $\Phi\left(D^{*}, e_{i}\right)$ we get that the above expression is equal to $y_{*}$. Therefore, it is optimal to reject $y<y_{*}$ and accept $y>y_{*}$, which is what the strategy $f_{*}$ suggests.

Q.E.D.

\section{AN ANALYSIS OF THE SOLUTION}

The main theorem established that the equilibrium agreement is such that type 1 agents get

$$
\begin{aligned}
& x_{*}=\frac{2(1-\delta)+\delta \alpha-\delta(1-\delta)(1-\alpha)(1-\beta)}{2(1-\delta)+\delta \alpha+\delta \beta} \text { or } \\
& y_{*}=\frac{\delta \alpha+(1-\delta) \delta(1-\alpha)(1-\beta)}{2(1-\delta)+\delta \alpha+\delta \beta}
\end{aligned}
$$

of the unit depending on the random selection of the proposer at each particular match. The equilibrium involves an absence of impasse: one partner makes an offer $x_{*}$ or $y_{*}$ which the other immediately accepts. Obviously, $x_{*}>y_{*}$ which reflects the bargaining advantage of being the first to propose. However, as we shall see shortly, the difference between $x_{*}$ and $y_{*}$ is negligible when the degree of impatience or the length of the period between bargaining sessions is small.

The value for a type 1 agent of participating in the market is

$$
V_{1}=\frac{\delta \alpha}{1-\delta+\delta \alpha}\left(\frac{1}{2} x_{*}+\frac{1}{2} y_{*}\right)=\frac{\delta \alpha}{2(1-\delta)+\delta \alpha+\delta \beta} .
$$


Similarly, for a type 2 agent it is

$$
V_{2}=\frac{\delta \beta}{1-\delta+\delta \beta}\left(\frac{1}{2}\left(1-x_{*}\right)+\frac{1}{2}\left(1-y_{*}\right)\right)=\frac{\delta \beta}{2(1-\delta)+\delta \alpha+\delta \beta} .
$$

In what follows we examine how the equilibrium values of $x_{*}$ and $V_{i}$ depend on the parameters of the model. Recall that what drives the agents to settle immediately rather than drag on with the negotiations are the facts that time is valued and that disagreement in bargaining involves a loss of time. There is a direct loss of time due to the period lost between consecutive bargaining sessions and an indirect loss of time due to the possibility of losing a partner and then having to wait for another opportunity. The equilibrium agreement depends on the relative importance of these two elements. They are captured in the model by the parameters $\delta, \alpha$, and $\beta$ which are exogenous throughout.

\section{The Role of $\delta$}

When the degree of impatience is small ( $\delta$ is close to 1 ), then the decisive forces are the respective market opportunities as represented by $\alpha$ and $\beta$, and the unit is divided approximately according to the relative sizes of $\alpha$ and $\beta$. To see this observe that

$$
\lim _{\delta \rightarrow 1} x_{*}=\lim _{\delta \rightarrow 1} y_{*}=\lim _{\delta \rightarrow 1} V_{1}=\frac{\alpha}{\alpha+\beta} .
$$

If, however, the degree of impatience is large ( $\delta$ is near 0 ), then the direct loss associated with one period's delay overshadows other considerations. In such a case the parties have approximately the same power and what determines each particular agreement is the random selection of the proposer. To see this observe that

$$
\lim _{\delta \rightarrow 0} x_{*}=1, \quad \lim _{\delta \rightarrow 0} y_{*}=0, \quad \lim _{\delta \rightarrow 0} V_{1}=0 .
$$

\section{The Length of a Bargaining Period}

The equilibrium agreement also depends on the significance of a bargaining period for the parties. To capture this point explicitly, let $\Delta$ denote the length of one period and let $\alpha(\Delta)=\alpha \Delta, \beta(\Delta)=\beta \Delta$, and $\delta(\Delta)=e^{-r \Delta}$, where $\alpha$ and $\beta$ are the arrival rates of new partners for the different types, and $r$ is the subjective rate of time preference. On substituting $\alpha(\Delta), \beta(\Delta)$, and $\delta(\Delta)$ into (5) and (6), one gets the equilibrium values as functions of $\Delta$. Since in many settings it is reasonable to assume that the length of one bargaining period is "small", let us consider the limiting equilibrium values as $\Delta \rightarrow 0$. Using l'Hôpital's rule we obtain

$$
\lim _{\Delta \rightarrow 0} x_{*}(\Delta)=\lim _{\Delta \rightarrow 0} y_{*}(\Delta)=\frac{r+\alpha}{2 r+\alpha+\beta}, \quad \lim _{\Delta \rightarrow 0} V_{1}(\Delta)=\frac{\alpha}{2 r+\alpha+\beta} .
$$


Thus, when the period between consecutive stages of the bargaining process is "short", the unit is divided approximately in the ratio $(r+\alpha):(r+\beta)$.

\section{The Agreement as a Function of $N_{1}$ and $N_{2}$}

Recall that the parameters $\alpha$ and $\beta$ are viewed as functions of $N_{1}$ and $N_{2}$ (the respective population sizes of type 1 and 2). The exact functional forms depend, of course, on the matching (or search) technology which was not modeled explicitly. Assume that this technology is such that the per-period overall rate of meetings, $M$, is fixed, ${ }^{6}$ and that all agents of a particular type are as likely to take part in one of the period's meetings. Thus, $\alpha(\Delta)=\left(M / N_{1}\right) \Delta$ and $\beta(\Delta)=$ $\left(M / N_{2}\right) \Delta$. On substituting these into (7) and (9) we get

$$
\begin{aligned}
\lim _{\delta \rightarrow 1} x_{*} & =\lim _{\delta \rightarrow 1} y_{*}=\frac{\left(M / N_{1}\right)}{\left(M / N_{1}\right)+\left(M / N_{2}\right)}=\frac{N_{2}}{N_{1}+N_{2}}, \\
\lim _{\Delta \rightarrow 0} x_{*} & =\lim _{\Delta \rightarrow 0} y_{*}=\frac{r+\left(M / N_{1}\right)}{2 r+\left(M / N_{1}\right)+\left(M / N_{2}\right)} \\
& =\frac{\left(r N_{1} N_{2} / M\right)+N_{2}}{\left(2 r N_{1} N_{2} / M\right)+N_{1}+N_{2}} .
\end{aligned}
$$

The relative sizes of $N_{1}$ and $N_{2}$ affect the equilibrium agreement in the expected direction: the greater is $N_{2}$ relative to $N_{1}$, the larger will be the equilibrium share of agents of type 1 . That is, members of the short side of the market have an advantage, but their advantage does not enable them to appropriate all the surplus.

\section{The Relations with Competitive Equilibrium}

The last observations suggest that the solution described above is very different from the competitive equilibrium outcome, despite the fact that the market under consideration has many of the basic properties which are thought to characterize a competitive environment. To clarify this statement consider the example in which type 1 agents are sellers and type 2 agents are buyers of an indivisible good. Each seller has one unit for sale and his reservation value is zero. Each buyer seeks to buy one unit and his reservation value is one. Let us maintain the steady state assumption that there are always $N_{i}$ agents of type $i$. The competitive equilibrium is a price that equates demand to supply. If in the example considered here $N_{2}>N_{1}$, then the competitive equilibrium price is 1 and the sellers (type 1) appropriate all the surplus. This result stands in sharp contrast to the equilibrium outcome in our model as captured by (10) and (11).

\footnotetext{
${ }^{6}$ There are several ways to relate $M$ to $N_{1}$ and $N_{2}$. One example is the linar search technology $M=k_{1} N_{1}+k_{2} N_{2}$, where $k_{i}$ is interpreted as the search intensity of type $i$ agents and hence $k_{i} N_{i}$ is interpreted as the total number of meetings initiated by type $i$ agents. Using such a representation one can also consider endogenous choice of the $k_{i}$ 's.
} 
It seems that this contrast points out a certain difficulty in the meaning of the concept of competitive equilibrium. To see this recall that the missing element from the description of a competitive market is the specification of the micromechanism which leads to the formation of prices. A standard textbook justification for the competitive equilibrium concept is that, under "competitive conditions" such as smallness of the agents and negligible transaction costs, reasonable mechanisms of price formation will lead approximately to the competitive equilibrium price. The model of this paper specifies a reasonable mechanism of price formation in the context of a market whose basic characteristics are very similar to those of a competitive market. The seeming difference is that, in the present model, the interaction among traders may involve time costs (in the event that an agreement is not reached immediately). However, it seems that any reasonable mechanism of price formation must involve some type of costs and the idea behind the concept of competitive equilibrium is that it approximately describes the outcome when such costs are made negligible. Yet, even in the limit of the present model, as the time costs are made negligible (i.e., $\delta \rightarrow 1$ or $\Delta \rightarrow 0$ ), the equilibrium result as given by (10) or (11) is quite different from the competitive equilibrium outcome described above.

The significant deviation from the competitive equilibrium (even when $\Delta$ is negligibly small) is closely related to the familiar point made by Diamond [3]. Diamond considers a market where the sellers set the prices, and each buyer knows the price distribution but can find out about a particular seller's price only by incurring a positive search cost $k$. The result is that, regardless of how small is $k$ (provided that $k>0$ ) and despite the presence of many sellers, the only perfect equilibrium price is the monopoly price. The intuitive argument follows from the observation that whenever the lowest price is lower than the monopoly price, the seller who charges this price can profit from slightly raising it (the price raise will not drive away any customers if it is small relative to $k$ ). Obviously, the result is due to the existence of buyers' search costs and due to the fact that sellers can commit themselves to prices while buyers cannot (if only buyers can commit themselves to prices and if the sellers incur the cost $k$, then the equilibrium price is the monopsony price).

The deviation from the competitive equilibrium in the present model is explained by the same elements which appear in a somewhat more complicated combination. Here both buyers and sellers can be in a position to commit themselves to a price but only for the duration of one period, and both sides incur costs of delay (search) which in this case are determined endogeneously. A similar argument to the one presented by Diamond shows that in any meeting the party who makes the offer (commits itself for one period) is able to appropriate the other party's cost of delay. But, in contrast to Diamond's model, this does not make the prices crawl to the monopoly (or monopsony) price, since because of the symmetric role of the parties, a party can reject an offer knowing that it has a chance to become a proposer in the next meeting. The balance is struck at the pair $x^{*}, y^{*}$ which has the property that each proposer takes full advantage of its rival's cost of delay. As in Diamond's model here too the extent of the 
deviation from the competitive equilibrium does not depend on the absolute size of the costs of delay (the length of the period) but rather on the existence of such positive costs. In fact, the average equilibrium agreement, $\frac{1}{2}\left(x^{*}+y^{*}\right)$, depends on the relative sizes of the parties' costs of delay, while the absolute sizes of these costs affect only the advantage, $x^{*}-y^{*}$, of being the proposer.

\section{The Relations with the Diamond-Mortensen Approach}

Finally, let us point out the relations between the equilibrium agreement in the present model and the equilibrium agreement of the type discussed in the above cited works of Diamond and Mortensen. In their models when two agents meet they agree immediately on an equal division of the difference between the unit and the sum of their reservation values. That is, they adopt Nash's bargaining solution. Let $(\bar{x}, 1-\bar{x})$ denote the equilibrium partition obtained in the present model when our strategic approach is replaced by Nash's bargaining solution, and let $\bar{V}_{i}, i=1,2$, denote the agents' reservation values in this case. At this equilibrium

$$
\begin{aligned}
& \bar{x}=\delta \bar{V}_{1}+\frac{1-\delta \bar{V}_{1}-\delta \bar{V}_{2}}{2}, \\
& 1-\bar{x}=\delta \bar{V}_{2}+\frac{1-\delta \bar{V}_{1}-\delta \bar{V}_{2}}{2}, \\
& \bar{V}_{1}=\alpha \bar{x}+(1-\alpha) \delta \bar{V}_{1}, \\
& \bar{V}_{2}=\beta(1-\bar{x})+(1-\beta) \delta \bar{V}_{2} .
\end{aligned}
$$

By solving this system we get the equilibrium agreement

$$
\bar{x}=\frac{1-\delta+\delta \alpha}{2(1-\delta)+\delta \alpha+\delta \beta} .
$$

Comparing $\bar{x}$ with the equilibrium agreements of our model, $x_{*}$ and $y_{*}$, we discover that

$$
\bar{x}=\frac{1}{2}\left(x_{*}+y_{*}\right), \quad \lim _{\delta \rightarrow 1} \bar{x}=\lim _{\delta \rightarrow 1} x_{*}, \quad \lim _{\Delta \rightarrow 0} \bar{x}=\lim _{\Delta \rightarrow 0} x_{*} .
$$

Thus, at the limits (as $\delta \rightarrow 1$ or $\Delta \rightarrow 0$ ), the axiomatic bargaining approach and the strategic approach result in the same equilibrium agreements. When the time value of one bargaining period has some significance, the equilibrium agreements differ, $x_{*}>\bar{x}>y_{*}$, but the values attached to participation in the market are still the same, $V_{i}=\bar{V}_{i}$.

The Hebrew University 


\section{REFERENCES}

[1] Binmore, K. G.: "Perfect-Equilibria in Bargaining Models," International Centre for Economics and Related Disciplines, London School of Economics, D.P. 82/58, 1982.

[2] Derman, C.: Finite State Markovian Decision Processes. New-York: Academic Press, 1970.

[3] Diamond, P. A. "A Model of Price Adjustment, Journal of Economic Theory, 3 (1971), 156-168.

[4] - "Mobility Costs, Frictional Unemployment and Efficiency," Journal of Political Economy, 89 (1981), 798-812.

[5] -Wage Determination and Efficiency in Search Equilibrium," Review of Economic Studies, 49 (1982), 217-227.

[6] Diamond, P. A., AND E. MASkin: "An Equilibrium Analysis of Search and Breach of Contract, I: Steady States," Bell Journal of Economics, 10 (1979), 282-316.

[7] Fishburn, P. C., AND A. Rubinstein: "Time Preference," International Economic Review, 23 (1982), 677-694.

[8] Mortensen, D. T.: "The Matching Process as a Noncooperative Bargaining Game," in The Economics of Information and Uncertainty, ed. by J. J. McCall. Chicago and London: The University of Chicago Press, 1982.

[9] __ : "Property Rights and Efficiency in Mating, Racing and Related Games," The American Economic Review, 72 (1982), 968-979.

[10] Rubinstein, A.: "Perfect Equilibrium in a Bargaining Model," Econometrica, 50 (1982), 97-109.

[11] Shaked, A., AND J. Sutton: "Involuntary Unemployment as a Perfect Equilibrium in a Bargaining Model," Econometrica, 52(1984), 1351-1362.

[12] Stahl, I.: Bargaining Theory. Stockholm: Stockholm School of Economics, 1972.

[13] Zusman, P., AND C. Bell: "The Equilibria Set of Dynamic Contracts," mimeo, The Hebrew University of Jerusalem, 1981. 